\title{
Falla hepática fulminante en niños
}

\author{
JORGE ROQUE E. ${ }^{1}$, GLORIA RÍOS M. ${ }^{1}$, CONSTANZA PINOCHET V. ${ }^{2}$, \\ PAULINA VIGNOLO A. ${ }^{2}$, ROBERTO HUMERES A. ${ }^{1}$, HORACIO RÍOS R. ${ }^{1}$, \\ MARTA QUIROGA G. ${ }^{1}$, MARÍA GABRIELA RODRÍGUEZ M. ${ }^{1}$, \\ XIMENA MORA ${ }^{1}$, MONTSERRAT RIUS A. ${ }^{1,3}$, JUAN HEPP K. ${ }^{1}$ \\ 1. Unidad de Trasplante, Clínica Alemana de Santiago y Facultad de Medicina Universidad del Desarrollo. Santiago de Chile. \\ 2. Interna $7^{\circ}$ año de medicina, Universidad del Desarrollo. \\ 3. Enfermera Universitaria.
}

\begin{abstract}
Acute liver failure in children. Experience of a liver transplant center

Background: Acute liver failure (ALF) in childhood is defined as biochemical evidence of liver injury, absence of known chronic liver disease and coagulopathy not corrected by vitamin $\mathrm{K}$ administration, with INR greater than 1.5 if the patient has encephalopathy or greater than 2.0 if the patient does not have encephalopathy. Objective: Report the experience of a single liver transplant center (LT) in the treatment of 8 children with ALF and review the literature. Method: Retrospective review of clinical charts of patients with ALF. Results: The median age was 8 years-old (range 0-11), three females. Five patients underwent LT. Two patients died, one of them LT. The etiologies were 4 undetermined, 1 autoimmune, 1 Wilson Disease, 1 Parvovirus and 1 chronic graft rejection. All grafts were from cadaver donor, 3 of them reduced. Two out of five patients with encephalopathy grade III-IV died. The one year survival rate was $75 \%$. Conclusions: Children with ALF should be treated in experienced centers with facilities for liver transplant. Transplantation should be offered only if the underlying disease is treatable by liver replacement and if transplant prognosis is better than that of the underlying disease.

(Key words: acute liver failure, liver transplant).

Rev Chil Pediatr 2009; 80 (2): 144-149
\end{abstract}

\section{RESUMEN}

Falla hepática fulminante (FHF) en la infancia se define como evidencia bioquímica de daño hepático, sin antecedentes conocidos de enfermedad hepática crónica, coagulopatía no corregida por la administración de vitamina $\mathrm{K}$ e INR superior a 1,5 si el paciente tiene encefalopatía o superior a 2 si no tiene encefalopatía. Objetivo: Presentar la experiencia de un centro de trasplante hepático (TH) en el tratamiento de 8 niños con FHF y revisar la literatura. Pacientes y Método: Revisión retrospectiva de la historia clínica de pacientes con FHF. Resultados: La edad media fue de 8 años, rango 0-11, tres sexo femenino. Cinco pacientes fueron

Trabajo recibido el 29 de agosto de 2008, devuelto para corregir el 12 de enero de 2009, segunda versión el 02 de febrero de 2009, aceptado para publicación el 16 de febrero de 2009.

Correspondencia a:

Dr. Jorge Roque E.

E-mail: jroque@alemana.cl 
sometidos a TH. Dos pacientes fallecieron, uno de ellos con TH. La etiología fue indeterminada en 4 pacientes, 1 autoinmune, 1 enfermedad de Wilson, 1 parvovirus y 1 rechazo crónico del injerto. Todos los injertos fueron de donante cadáver, 3 de ellos reducidos. Dos de cinco pacientes con encefalopatía grado III-IV fallecieron. La tasa de sobrevida al año fue de 75\%. Conclusión: El manejo de la FHF debe realizarse en un centro con capacidad de realizar $\mathrm{TH}$, aunque no todos los pacientes requerirán finalmente esta terapia. El TH debiera ser ofrecido sólo si la enfermedad subyacente es tratable con reemplazo hepático y si el pronóstico del TH es mejor que el de la enfermedad misma.

(Palabras clave: Falla hepática fulminante, trasplante hepático).

Rev Chil Pediatr 2009; 80 (2): 144-149

\section{Introducción}

La falla hepática fulminante (FHF) es una entidad clínica caracterizada por aparición de súbito daño hepático grave en un paciente sin historia conocida de enfermedad hepática crónica, coagulopatía que no se corrige con administración de vitamina K e INR mayor de 1,5 en presencia de encefalopatía o mayor de 2 sin encefalopatía, haciendo la salvedad, que la encefalopatía hepática (EH) en lactantes y niños pequeños es difícil de objetivar, por lo que su presencia no es requisito para diagnosticar la FHF a esas edades ${ }^{1,2}$.

En USA cada año se someten a trasplante hepático (TH) 2500 adultos, siendo el $6 \%$ por FHF y representando en niños el $11 \%$ de todos los TH pediátricos ${ }^{3}$. En Chile, un reporte en 132 niños sometidos a $\mathrm{TH}$, refiere que el $20,4 \%$ fue por $\mathrm{FHF}^{4}$, mientras que en Argentina el $30 \%$ de los $\mathrm{TH}$ pediátricos serían por $\mathrm{FHF}^{5}$. Afecta por igual a niños de ambos sexos, presentando tendencia a una mayor incidencia en lactantes menores y adolescentes ${ }^{1,6}$.

Las causas de FHF en niños son variadas y dependen fundamentalmente de la edad y del área geográfica que se evalúe, es así como en países en vías de desarrollo la causa más frecuente es la infección por virus hepatitis A (VHA) y posiblemente E, mientras que en países desarrollados es la intoxicación medicamentosa, principalmente por Acetoaminofeno ${ }^{1,2,4,6,7}$. Otras etiologías descritas son las autoinmunes, isquemicas-vasculares, metabólicas, otras infecciones virales y misceláneas. Hasta un $50 \%$ permanecen desconocidas ${ }^{1,2,6,7,8}$.

La mortalidad de la FHF en la era previa al TH alcanzaba el $80 \%{ }^{9,10}$, sin embargo, la sobrevida a un año plazo mejoró dramáticamente con la introducción del TH como terapia de emergencia a aproximadamente $70-90 \% 0^{1-5,9,11,12}$.

El objetivo de este artículo es reportar la experiencia de nuestro centro en el tratamiento de 8 pacientes pediátricos ingresados con diagnóstico de FHF, 5 de los cuales fueron sometidos a TH de emergencia, así como revisar el estado actual de la literatura.

\section{Pacientes y Método}

Se revisó retrospectivamente las fichas clínicas de los pacientes pediátricos ingresados a nuestro servicio con el diagnóstico de FHF entre mayo 1997 y abril 2008. La recolección de datos se realizó en una plantilla Excel ${ }^{\circledR}$ diseñada para este trabajo.

Todos los pacientes recibieron tratamiento general para FHF según lo descrito por Nadalin et $\mathrm{al}^{13}$, lo cual no ha cambiado significativamente en la última década.

El estudio etiológico de rutina se basó en la determinación de IgM VHA, Antígeno HBs, PCR para virus hepatitis C (VHC), IgM CMV, IgM Epstein Barr(VCA), IgM y PCR parvovirus B19 (HPV B19), anticuerpos antinucleares, anticuerpos antimúsculo liso, anticuerpos anti LKM y ceruloplasmina. Además fueron evaluados por oftalmólogo, neurólogo infantil, hepatólogo, infectólogo, equipo de trasplante hepático e intensivistas pediátricos. También se realizó ecografía doppler abdominal, exámenes de rutina de laboratorio y radiológicos en la unidad de cuidados intensivos de pediatría (UCIP) y cualquier otro examen que fuera considerado necesario por alguno de los especialistas. Todos los pacientes fueron ingresados a la UCIP. 
La indicación de TH se basó en la evolución clínica de los pacientes, considerando la etiología, el grado de encefalopatía y la edad, en concomitancia con la aplicación de los criterios de King's College ${ }^{3,8,11}$.

Las contraindicaciones al TH fueron daño cerebral irreversible, fallo multiorgánico e infección no controlada ${ }^{11,13}$.

El diagnóstico de rechazo agudo se realizó mediante biopsia hepática ante sospecha por empeoramiento de bioquímica hepática en sangre.

\section{Resultados}

De los 8 pacientes, 5 fueron derivados de otros centros hospitalarios ante la posibilidad de necesitar TH de emergencia. En la tabla 1 se resumen sus principales características clínicas. En los pacientes sometidos a TH, en que no hubo concordancia entre el peso del donante y del receptor, se realizó reducción del injerto antes del implante. En 3 pacientes con fallo renal agudo, se realizó terapia de reemplazo renal, ya sea con hemofiltración y/o hemodiálisis. La indicación de ventilación mecánica (VM) en los 3 primeros pacientes y el séptimo, fue por encefalopatía. Los otros 2 que se trasplantaron sólo requirieron la VM menos de 24 horas post $\mathrm{TH}$. Todos los pacientes fueron activados como urgencia para $\mathrm{TH}$ a nivel nacional, pero en 2 la enfermedad mejoró (pacientes 4 y 8) y otro presentó daño cerebral irreversible antes del $\mathrm{TH}$, por lo que fueron retirados de la lista de urgencia. Un paciente fue sometido a $2 \mathrm{TH}$, debido a que perdió el primer injerto por presentar un shock séptico grave por germen gram negativo, en quién se suspendió la inmunosupresión, presentando una disfunción con rechazo agudo del injerto grave e irreversible ${ }^{14}$. El octavo paciente fue un recién nacido con antecedentes de un hermano fallecido a los 45 días de vida por FHF de etiología desconocida. El paciente presentó hipoglucemia de $12 \mathrm{mg} / \mathrm{dl}$ asociada a movimientos anormales, hiprotrombinemia grave que no corrigió con vitamina $\mathrm{K}$ y alteración de las pruebas hepáticas sanguíneas. $\mathrm{Su}$ electroencefalograma y resonancia nuclear magnética de cerebro fueron normales. El estudió etiológico no fue concluyente para enfermedades metabólicas, infecciosas, malformaciones y lupus. Sin embargo, presentó niveles altos de ferritina, $673 \mathrm{ng} / \mathrm{ml}$ (valor normal 7-140 $\mathrm{ng} / \mathrm{ml}$ ), lo cual hizo sospechar una hemocromatosis neonatal ${ }^{11,15}$, que no pudo confirmarse, ya que también es un reactante de fase aguda y sus niveles fueron progresivamente normalizándose, al igual que la condición del paciente hasta su alta. No se realizó biopsia hepática ni de glándulas salivales.

Cuatro de los 5 pacientes sometidos a $\mathrm{TH}$

Tabla 1. Principales características clínicas de los pacientes pediátricos con FHF

\begin{tabular}{lcccccccc}
\hline Paciente & $\mathbf{1}$ & $\mathbf{2}$ & $\mathbf{3}$ & $\mathbf{4}$ & $\mathbf{5}$ & $\mathbf{6}$ & $\mathbf{7}$ & $\mathbf{8}$ \\
\hline Edad (años) & 7 & 10 & 7 & 8 & 11 & 9 & 13 & 0 \\
Sexo & $\mathrm{M}$ & $\mathrm{F}$ & $\mathrm{F}$ & $\mathrm{F}$ & $\mathrm{M}$ & $\mathrm{M}$ & $\mathrm{M}$ & $\mathrm{M}$ \\
TH & $\mathrm{si}$ & $\mathrm{si}$ & $\mathrm{si}$ & no & si & si & no & no \\
No TH & 1 & 1 & 2 & 0 & 1 & 1 & 0 & 0 \\
Donante & cadáver & cadáver & cadáver & no & cadáver & cadáver & no & no \\
Injerto & reducido & completo & reducido & no & completo & reducido & no & no \\
Encefalopatía & IV & III & III & II & I & III & IV & I \\
Etiología & IDP & IDP & PVB19 & Al & Wilson & IDP & RC, DGI & IDP \\
Falla renal & si & si & si & no & no & no & no & no \\
Días VM & 21 & 17 & 10 & no & 1 & 1 & 6 & no \\
Vivo & si & no & si & si & si & si & no & si \\
Tiempo Hosp & 79 días & 32 días & 44 días & 17 días & 13 días & 16 días & 24 días & 21 días \\
RCA & no & si & si & no & si & si & no & no \\
TS (meses) & 132 & 1 & 64 & 52 & 41 & 32 & 1 & 20 \\
\hline
\end{tabular}

FHF: falla hepática fulminante; M: masculino; F: femenino; TH: trasplante hepático; IDP: idiopático; RC: rechazo crónico; DGI: disfunción grave del injerto; PVB 19: parvovirus B19; Al: autoinmune; VM: ventilación mecánica; Hosp: hospitalización; RCA: rechazo celular agudo; TS: tiempo seguimiento. 
presentaron rechazo celular agudo en el período post operatorio, los cuales se resolvieron satisfactoriamente con tratamiento esteroidal.

\section{Discusión}

Siete de nuestros pacientes eran de edad escolar entre 7 y 11 años, lo que contrasta con lo descrito en la literatura que identifica 2 picos etarios, lactantes menores y adolescentes ${ }^{1,6,16}$. El número pequeño de la muestra podría explicar este fenómeno.

Es sabido que la $\mathrm{EH}$ no es un requisito absoluto para establecer el diagnóstico de FHF en lactantes y niños menores ${ }^{1,2,12}$, pero sí se relaciona con el pronóstico ${ }^{10,11,16}$. Squires et al, en un estudio de 348 pacientes con FHF reporta que los pacientes que no presentaron $\mathrm{EH}$ tenían mayor posibilidad de resolución espontánea del cuadro que los que sí la habían presentado $^{1}$. En nuestra serie 5 pacientes presentaron $\mathrm{EH}$ grado III o IV, 2 de ellos fallecieron, en cambio los 3 que tuvieron EH I o II sobrevivieron.

El $50 \%$ de los casos fueron de etiología indeterminada lo que coincide con lo reportado por la mayoría en Europa y Norteamérica (tabla 2). En cambio, este porcentaje es menor en los países latinoamericanos, creemos que debido a que el VHA corresponde a una de las principales causas de FHF en niños ${ }^{6,7}$, hecho que no se refleja en nuestra experiencia.

El conocer la etiología no es un hecho menor, ya que se relaciona con la terapéutica y la sobrevida de los pacientes. Es así como, pacientes con FHF debido a intoxicación por paracetamol presentan una evolución más aguda y benigna en el tiempo y con mayor porcentaje de recuperación espontánea en relación a otras etiologías ${ }^{1,17}$. Un estudio de prevalencia nacional realizado en EEUU, reporta en pacientes con FHF por intoxicación con paracetamol 68\% de sobrevida sin necesidad de TH, con sólo un 6\% de ellos que debió ser sometido a TH, versus lo ocurrido en los casos de FHF por reacción idiosincrásica a drogas donde sólo el $25 \%$ presentó sobrevida espontánea y un $53 \%$ necesitó ser trasplantado. El mismo estudio refiere que además de la intoxicación por paracetamol, las otras etiologías de FHF que tienen buena sobrevida con menos probabilidad de necesitar TH son shock y VHA ${ }^{16}$.

La tasa de sobrevida para los pacientes sometidos a TH depende además de otros factores, como la edad del paciente, la curva de aprendizaje del equipo que realiza el TH y del número de trasplantes requeridos por el receptor $^{9,11}$. Cochran et al, reportan que pacientes menores de 1 año tuvieron una sobrevida a 10 años plazo de $65 \%$ comparado con una de $79 \%$ para niños mayores ${ }^{11}$. Si bien, a mayor número

Tabla 2. Etiología de la FHF en niños alrededor del mundo. Cifras expresadas en porcentajes del número total de pacientes de cada serie

\begin{tabular}{|c|c|c|c|c|c|c|c|}
\hline Causas FHF & $\begin{array}{l}\text { Alegría } \\
\text { y cols }{ }^{21}\end{array}$ & $\begin{array}{l}\text { Squires } \\
\text { et al }{ }^{1}\end{array}$ & $\begin{array}{l}\text { Lee } \\
\text { et } \mathrm{al}^{20}\end{array}$ & $\begin{aligned} & \text { Cochra } \\
< & 1 \text { año }\end{aligned}$ & $\begin{array}{l}\text { et al. }{ }^{11} \\
>1 \text { año }\end{array}$ & $\begin{array}{c}\text { Montijo- } \\
\text { Barrios } \\
\text { et al }\end{array}$ & $\begin{array}{c}\text { Cuarterolo } \\
\text { et } \mathrm{al}^{5}\end{array}$ \\
\hline $\begin{array}{l}\text { Número total de pacientes } \\
\text { de la serie }\end{array}$ & 12 & 348 & 97 & 80 & 146 & 30 & 50 \\
\hline Infecciosas totales & 33,4 & 6 & 54,6 & $15\left(^{*}\right)$ & 41 & 63,3 & 68 \\
\hline Hepatitis A & 16,7 & 1 & 9 & & 10 & 40 & 68 \\
\hline Otros virus $(* *)$ & 16,7 & 5 & 39 & & 31 & 13,3 & 0 \\
\hline Otras infecciones & 0 & 0 & 6,6 & & 0 & 10 & 0 \\
\hline Auto inmune & 8,3 & 6 & 3,1 & 0 & & 13,3 & 2 \\
\hline Drogas & 8,3 & 19 & 19,6 & 0 & 10 & 3,5 & 2 \\
\hline Indeterminado & 41,7 & 49 & 0 & 16 & 47 & 13,3 & 28 \\
\hline Metabólicas & 0 & 10 & 22,7 & $58(\#)$ & & 6,6 & 0 \\
\hline Otras causas $\left({ }^{a}\right)$ & 8,3 & 10 & 0 & 10 & 2 & 0 & 0 \\
\hline
\end{tabular}

FHF: falla hepática fulminante. $\left(^{*}\right)$ Hepatitis virales no definidas. $\left(^{* *}\right)$ Hepatitis B, hepatitis C, hepatitis NoA-NoE, Adenovirus, Citomegalovirus, Epstien Barr, Enterovirus, Herpes simple, Parvovirus B19. (\#) Hemocromatosis neonatal $16 \%$ y $42 \%$ otras enfermedades metabólicas. (a) Budd-Chiari, leucemia, enfermedad veno-oclusiva, síndrome hemofagocítico, shock. 
Tabla 3. Sensibilidad, especificidad, VPP, VPN y ABC de cuatro parámetros pronósticos que fueron capaces de definir sobrevida o muerte sin TH, usando un análisis de doble proporción en 44 niños con FHF, según Dhawan et al ${ }^{17}$

\begin{tabular}{lccccc} 
& Sensibilidad & Especificidad & VPP & VPN & ABC \\
\hline INR $\geq 4$ & 86 & 73 & 73 & 14 & 0,79 \\
Bilirrubina $\geq 235 \mu$ mol/L $\left(^{*}\right)$ & 85 & 65 & 79 & 73 & 0,76 \\
Edad mayor de 2 años & 93 & 52 & 50 & 94 & 0,74 \\
Más de $9 \times 10^{9} /$ leucocitos & 89 & 71 & 83 & 80 & 0,78 \\
\hline
\end{tabular}

VPP: valor predictivo positivo; VPN: valor predictivo negativo; ABC: área bajo la curva. ${ }^{*}$ ) $13,8 \mathrm{mg} / \mathrm{dl}$

de TH sometido el receptor, mayor es su morbimortalidad, nosotros tuvimos un caso de FHF por PVB19 que requirió 2 TH y que no falleció, aunque si tuvo mayor morbilidad, pero finalmente se incorporó a su vida normal.

En nuestro estudio 5 de 8 pacientes $(63 \%)$ fueron trasplantados y de éstos sólo uno falleció, lo que a pesar del número reducido de casos concuerda con lo descrito previamen$\mathrm{te}^{2,5,9,16}$. Esto demostraría que un centro pequeño también puede tener buenos resultados en el tratamiento del FHF en niños.

La paciente 4 con FHF debida a hepatitis autoinmune (HAI), sobrevivió sin requerir TH. Ella estuvo activada como urgencia para $\mathrm{TH}$, sin embargo, debido a antecedentes familiares de autoinmunidad y tratarse de un paciente sexo femenino, se sospechó HAI, realizándose un tratamiento de prueba con esteroides, observando clara mejoría de sus parámetros de insuficiencia hepática. En esta presentación clínica los auto anticuerpos estaban ausentes y la biopsia hepática demostró inflamación portal y lobulillar, con actividad de interfase y necrosis confluente, lo que según Bucuvalas et al, es característico de esta etiología en su presentación aguda ${ }^{2}$. En su evolución, 5 meses después presentó aplasia medular severa que se asocia frecuentemente a HAI, manejándose con azathioprina y ciclosporina $\mathrm{A}$, manteniendo una buena función hepática.

Uno de los grandes dilemas en cuanto al manejo de la FHF en niños es cuando poner a estos en la lista de trasplante para que puedan recibir el injerto en forma oportuna. Por un lado se podría esperar la recuperación espontánea, sin embargo, se corre el riesgo de llegar demasiado tarde al $\mathrm{TH}$ con la consiguiente mortalidad, y por otro lado, el someter a un niño a TH implica otra morbimortalidad. En nuestro centro nos guiamos con los criterios de King's College, aun vigentes en la actualidad ${ }^{3,8,11,12,16}$. Sin embargo, existen muchos criterios reportados para evaluar el pronóstico y la necesidad de $\mathrm{TH}$, por ejemplo el INR, bilirrubina, edad mayor de 2 años y recuento de leucocitos se reportan recientemente como predictores de mal pronóstico de FHF sin TH según Dhawan et al. comparando 29 niños fallecidos versus 15 no fallecidos por FHF no sometidos a TH (tabla $3)^{17}$. En otro estudio realizado en Alemania, con 17 niños sometidos a TH por FHF, se reportó que se utilizaron como criterios para indicar $\mathrm{TH}$, la presencia de $\mathrm{EH} \geq$ grado III, INR $>2$, bilirrubina $>18 \mathrm{mg} / \mathrm{dl}$, fuerte tendencia a la hipoglucemia y reducción del tamaño del hígado monitorizado por ecografía, logrando una sobrevida de los pacientes sometidos a TH a 5 años de $94 \%{ }^{13}$. De todas maneras, aunque no existen criterios universalmente aceptados de indicación de $\mathrm{TH}^{17}$, se recomienda trasladar al paciente con FHF que cursa con EH de rápida progresión ( 0 a 3-4 en 3 días) a un centro con capacidad de realizar TH, ya que en estos casos existe alta probabilidad de muerte $\sin$ $\mathrm{TH}^{11}$.

El escaso número de injertos hepáticos de donante de cadáver disponibles en tiempo oportuno, a obligado a desarrollar nuevas técnicas para ampliar su disponibilidad, siendo los más usados en la actualidad el split liver o bipartición del hígado, que permite beneficiar a dos receptores simultáneamente, y la obtención de un segmento hepático de un donante vivo ${ }^{1,2,5,12,11,13}$. Nosotros no hemos necesitado hasta el momento de estas técnicas.

Últimamente se han desarrollado nuevos procedimientos que buscan remover de la circula- 
ción las toxinas involucradas en el FHF, y así permitirle al paciente ganar tiempo vital a la espera de que su hígado se recupere o bien pueda ser sometido a TH en las mejores condiciones posibles. Entre estos se cuentan los dispositivos bioartificiales, de los cuales la terapia hepática extracorporea (ELAD) que utiliza líneas celulares de hepatoblastoma humano alojados en un cartucho y el hígado bioartificial (BAL) que utiliza hepatocitos de porcino son los ejemplos más reportados ${ }^{11,13}$.

El sistema de diálisis de toxinas hepáticas mediante la recirculación de adsorbentes molecular (MARS) es el más novedoso de los sistemas artificiales de depuración hepática ${ }^{18}$. Un meta análisis de Tissieres et al, reportó que si bien el MARS parecía ser un arma terapéutica prometedora en la FHF, no existían suficientes datos en la literatura que justificaran su uso en niños ${ }^{19}$. Sin embargo, un óptimo manejo preoperatorio en una UCIP con experiencia en estos casos, debe incluir el MARS como una de sus alternativas terapéuticas en casos bien determinados ${ }^{11,17}$.

En resumen, se presenta una serie de 8 pacientes pediátricos con $\mathrm{FHF}, 5$ de los cuales fueron sometidos a $\mathrm{TH}$, con una sobrevida global de $75 \%$ al año de seguimiento. El manejo de la FHF debe realizarse en un centro con capacidad de realizar $\mathrm{TH}$, aunque no todos los pacientes requerirán finalmente esta terapia. E1 TH debiera ser ofrecido sólo si la enfermedad subyacente es tratable con reemplazo hepático y si el pronóstico del $\mathrm{TH}$ es mejor que el de la enfermedad misma.

\section{Referencias}

1.- Squires R, Schneider B, Bucuvalas J, et al: Acute liver failure in children: The first 348 patients in the pediatric acute liver failure study group. J Pediatr 2006; 148 (5): 652-8.

2.- Bucuvalas J, Yazigi N, Squires R: Acute Liver Failure in Children. Clin Liver Dis 2006; 10 (1): 149-68.

3.- Krasko A, Deshpande K, Bonvino S: Liver failure, transplantation, and critical care. Crit Care Clin 2003; 19 (2): 155-83.

4.- Uribe $M$, Buckel E, Ferrario $M$, et al: Pediatric Liver Transplantation: Ten Years of Experience in a Multicentric Program in Chile. Transp Proc 2005; 37: 3375-7.
5.- Cuarterolo M, Ciocca M, López S, et al: Evolución de niños post-trasplante hepático luego del primer año de sobrevida. Medicina 2005, 65: 402-8.

6.- Montijo Barrios E, García López R, Cervantes Bustamante $R$, et al: Etiología de hepatitis fulminante en niños. Rev Enfer Infec Pediatr 2006; XX (77): 810.

7.- Ciocca M, Moreira-Silva SF, Alegria S, et al: Hepatitis A as an etiologic agent of acute liver failure in Latin America. Pediatr Infect Dis J 2007; 26: 711-5.

8.- Poniachik J, Quera R, Lui A: Insuficiencia hepática fulminante. Rev Méd Chil 2002; 130: 691-8.

9.- Durand P, Debray D, Mandel R, et al: Acute liver failure in infancy: A 14-year experience of a pediatric liver transplantation center. J Pediatric 2001; 139: 871-6.

10.- Novelli G, Rossi M, Pretagostini R, et al: MARS (Molecular Adsorbent Recirculating System): experience in 34 cases of acute liver failure. Liver 2002; 22: 43-7.

11.- Cochran JB, Losek JB: Acute Liver Failure in children. Pediatr Emerg Care 2007; 23 (2): 129-35.

12.- Kerkar N, Emre $S$ : Issues unique to pediatric liver transplantation. Clin Liver Dis 2007; 11: 323-35.

13.- Nadalin $S$, Heuer $M$, Wallot $M$, et al: Paediatric acute liver failure and transplantation: The University of Essen experience. Transpl Int 2007; 20 (6): 519-27.

14.- Roque EJ, Ríos $M G$, Hepp KJ, et al: Falla hepática fulminante por parvovirus B 19 y trasplante hepático, caso clínico. Rev Chil Pediatr 2005; 76 (4): 393 6.

15.- Grabhorn E, Richter A, Burdelski M, Rogiers X, Ganschow R: Neonatal hemochromatosis: long-term experience with favorable outcome. Pediatrics 2006; 118 (5): 2060-5.

16.- Lee WM: Acute liver Failure in the United States. Seminars in liver disease 2003; 23 (3): 217-26.

17.- Dhawan A, Cheesman P, Mieli-Vergani G: Approaches to acute liver failure in children. Pediatric Transplantation 2004; 8: 584-8.

18.- Contreras J, Poniachik J, Oksenberg D, et al: Diálisis con albúmina MARS (Molecular Adsorbent Recirculating System) como puente para el trasplante hepático en insuficiencia hepática fulminante: Presentación de tres casos. Rev Méd Chile 2004; 132 (5): 601-7.

19.- Sasbon JS, Devictor D, Tissieres P: Liver support for fulminant hepatic failure: Is it time to use the Molecular Adsorbents Recycling System in children? Pediatr Crit Care Medicine 2005; 6 (5): 585-91.

20.- Lee WS, McKiernan P, Kelly DA: Etiology, outcome and prognostic indicators of childhood fulminant hepatic failure in the United Kingdom. J Pediatr Gastroenterol Nutr 2005; 40 (5): 575-81.

21.- Alegría S, Ríos $G$, Gelcich A, et al: Estudio etiológico de falla hepática fulminante en niños en la región metropolitana. Resúmenes del XLIV Congreso chileno de pediatría. Rev Chil Pediatr 2004; 75 (6): 565 641 . 